\title{
Power consumption and size minimization of a wireless sensor node in automation system application
}

\begin{abstract}
In developing Wireless Sensor Node (WSN) especially in automation applications, the power consumption and the size minimization are the main concern. Therefore, some strategies have been proposed for investigating the power consumption in this field. These strategies provide knowledge to expect the WSN cost, complexity, and lifetime, as well as to provide recommendations to automation designers and enhance to optimize the energy consumed based on the applications. This paper proposes a wireless sensor measurement as a precise strategy for power consumption evaluation, the proposed method is less not only in cost, but also in terms of complexity, the paper presents an approach for evaluating the power consumption of a wireless sensor node in automation applications by attempting on experimental measurements along with a set of tools to automate the proposed approach. Starting from a MATLAB programming language code, we develop algorithms and node configuration in such a way that the analogue sensor signal, the processing and analysis area accomplished without using microcontroller, but only the XBee RF modules with 1+0.3 mWatt power consumption. In order to evaluate the proposed approach, we compare the results obtained by this proposed method against ones obtained through oscilloscope reading.
\end{abstract}

Keyword: Wireless sensor networks; Power consumption; Wireless data transmission rate; Wireless automation system 